Results $16.2 \%$ of patients at UHSM did not show response to omalizumab at 16 weeks. Baseline serum IgE levels in the nonresponse group were on average $77.28 \mathrm{kU} / \mathrm{L}$ lower than those in the response group, statistical analysis of the two groups show that this difference was significant $(P=0.04)$. Mean eosinophils in the true non-responder group were actually higher than those in the true responder group, however this difference was not statistically significant. No other demographic or disease specific measures predicted a lack of response to omalizumab.

Discussion The results from the study indicate that a lower baseline serum IgE may predict non-response to treatment with omalizumab. The results also show that non-response rates at the NWLC were lower than those demonstrated in clinical trials (INNOVATE), were consistent with other real life studies (PERSIST/APEX I and APEX II) but markedly lower than those quoted in the eXpeRience registry.

\section{P241 EOSINOPHIL APOPTOSIS IS NEGATIVELY ASSOCIATED WITH BODY MASS INDEX IN ASTHMA}

${ }^{1} \mathrm{~A}$ Thavakumar, ${ }^{2} \mathrm{AKA}$ Wright, ${ }^{1} \mathrm{MA}$ Ghebre, ${ }^{2} \mathrm{~T}$ Thornton, ${ }^{1,2} \mathrm{CE}$ Brightling. ${ }^{1}$ Department of Infection, Immunity and Inflammation, University of Leicester, Leicestershire, UK; ${ }^{2}$ Institute of Lung Health, NIHR Leicester Respiratory Biomedical Unit, University Hospitals of Leicester NHS Trust, Leicestershire, UK

\subsection{6/thoraxjnl-2016-209333.384}

Background Obese asthmatics are known to have reduced eosinophils in sputum, as well as poor control of asthma symptoms. ${ }^{1}$ We have shown that, compared to non-obese patients, there is an elevated number of eosinophils in the airway submucosa of obese asthmatic patients. ${ }^{2}$ This study aims to determine whether a differential susceptibility to apoptosis, between obese and non-obese patients, could contribute to these clinical observations.

Method Patients with a clinical diagnosis of asthma were recruited $(n=28)$ and consented at Glenfield Hospital for blood donation to study eosinophil apoptosis; the patients recruited had varying severities of asthma and BMI. Eosinophils were isolated from whole blood by negative immunomagnetic selection using CD16 microbeads to a purity of mean \pm SD 95.7\% ( \pm 4$)$. Purified eosinophils (Time 0) were placed into culture in RPMI (1640 + GlutaMAX-1 supplemented with 10\% FBS and 1\% penicillin and streptomycin) and harvested at 17 and 21 hours later to measure apoptosis by flow cytometry using Annexin $\mathrm{V}$ and Propidium Iodide (Becton Dickinson). Cells were considered apoptotic if they were Annexin $\mathrm{V}$ positive/PI negative and reported as a percentage of total eosinophils.

Results At 0 hours, the mean $\%$ of annexin $\mathrm{V}$ positive cells was $0.47 \%$ and there was no significant association with BMI $(\mathrm{r}=-0247, \mathrm{p}$ value $=0.245)$. At 17 and 21 hours there were $12.68 \%$ and $21.0 \%$ annexin $\mathrm{V}$ positive cells, respectively, and we noted a significant negative Pearson's correlation between eosinophil apoptosis and BMI at time $17(\mathrm{r}=-0.449 ; \mathrm{p}=0.028)$ and time $21(\mathrm{r}=-0.448 ; \mathrm{p}=0.028)$. These correlations were independent of lung function, steroid medication and percentage eosinophil purity.

Conclusion Eosinophils from obese asthmatic patients are less susceptible to apoptosis compared to those from non-obese patients. This may contribute to the differential presence of eosinophils in the lamina propria and airway of obese patients compared to non-obese individuals.

\section{REFERENCES}

1 Haldar P, Pavord ID, Shaw DE, et al. Cluster analysis and clinical asthma phenotypes. Am J Respir Crit Care Med 2008;178(3):218-224.

2 Desai D, Newby C, Symon FA, et al. Elevated sputum interleukin-5 and submucosal eosinophilia in obese individuals with severe asthma. Am J Respir Crit Care Med 2013;188(6):657-663.

\section{P242 THE AIRWAY MICROBIOTA IN HUMAN RHINOVIRUS INDUCED ASTHMA EXACERBATION}

EHC Wong, J Dhariwal, L Cuthbertson, P James, M Cox, M Moffatt, W Cookson, S Johnston. National Heart and Lung Institute, Imperial College London, London, UK

\subsection{6/thoraxjn-2016-209333.385}

Background Acute asthma exacerbations (AEs) cause significant morbidity. Up to $60 \%$ of AEs may be associated with respiratory viral infections, particularly human rhinoviruses (HRVs). The role of bacteria in AEs is unclear, yet antibiotics are frequently prescribed. Recent studies have demonstrated a greater abundance of potentially pathogenic bacteria (e.g. Haemophilus spp.) within the airway microbiota in asthma, whilst a greater abundance of commensals (e.g. Prevotella spp.) were observed in health. The aim of this study was to examine the changes within the airway microbiota in asthma in the context of a HRV-induced $\mathrm{AE}$ and evaluate if such changes correlate with clinical symptoms and lung function changes.

Methods Eleven moderate asthmatic (BTS step 3-4) and 12 healthy subjects were experimentally infected with HRV-16 and bronchoscopy was performed at baseline, 3 and 8 days following HRV-infection. Subjects completed daily symptom diary and spirometry. DNA was extracted from bronchoalveolar lavage and PCR amplification of the V3-V5 region of bacterial 16S rRNA gene was performed to evaluate microbiota community composition.

Results The microbiota composition did not significantly differ between healthy and asthmatic subjects at baseline, though healthy subjects exhibited significantly greater relative abundance of Prevotella spp. following HRV-infection ( $\mathrm{p}<0.05)$. At day 3 post-HRV infection, greater Prevotella spp. relative abundance was associated with lower symptom scores $(\mathrm{R} 2=0.56$, $\mathrm{p}<0.05)$. In contrast, at day 8 greater Neisseria spp. relative abundance was associated with greater peak flow decline $(\mathrm{R} 2=0.41, \mathrm{p}<0.05)$. Furthermore, HRV-16 viral load exhibited a significant linear relationship with the degree of microbiota community change (as measured by beta-diversity) $(\mathrm{R} 2=0.61$, $\mathrm{p}<0.05)$.

Conclusion Following HRV infection, greater Prevotella spp. relative abundance was associated with less symptoms whilst greater Neisseria spp. was associated with greater peak flow decline, suggesting an imbalanced microbiota may exacerbate airway inflammation and ultimately severity of AE. Viral load significantly correlated with degree of microbiota community change, implying HRV infection may directly perturb the airway microbiota. Further studies are needed to confirm these findings and explore the roles of Prevotella spp. and Neisseria spp. in exacerbating airway inflammation. 